\title{
A response time model for judging order relationship between two symbolic stimuli
}

\author{
R. PAGE, E. IZQUIERDO, A. SAAL, J. CODNIA, and C. EL HASI \\ Universidad Nacional de General Sarmiento, Buenos Aires, Argentina
}

\begin{abstract}
The response time to judge the order relationship between two symbolic stimuli is frequently modeled as the time spent in a (constant-rate) accumulative sampling process until a threshold is reached. We will show that empirical descriptions of observed effects in number comparisons suggest an accrual process that reaches the threshold at an exponential rate. The model accrual equations and stopping conditions have an immediate interpretation in terms of a simple quantitative connectionist network. The encoded stimuli and thresholds are inputs to the network. The former are considered to result from the participant's learning history, and the latter modulate the rearrangement of the network parts; each arrangement models a different task. We have found a good correlation between model predictions and other authors' experimental data, both in number comparisons and in experiments in which the ordering of the symbolic stimuli has been artificially induced. Incorrect answers are discussed, and predictions are compared with data. We will explore differences and similarities with other approaches, such as random walk and the symbolic comparison model. In a limit case, our model becomes identical to the discriminability model.
\end{abstract}

Since the late sixties (Moyer \& Landauer, 1967), a considerable amount of research has focused on the processes underlying observers' judgments of numerical magnitude. In this type of experiment, observers are asked to indicate which of two numbers is the larger (or smaller) or whether a random target number is larger or smaller than a certain fixed standard number. These experimental approaches are known as the selection and classification paradigms, respectively. The numbers are often single digits (1-9; Banks, Fujii, \& Kayra-Stuart, 1976; Moyer \& Landauer, 1967), but sometimes twodigit numbers have been employed (Dehaene, 1989, 1990; Hinrichs, Yurko, \& Hu, 1981; Link, 1990). In other, similar experiments, such as those involving the linear syllogistic reasoning task (Leth-Steensen \& Marley, 2000; Trabasso, Riley, \& Wilson, 1975), observers first learn an arbitrary order relationship between symbols within a small set, and are then presented with the symbols instead of the numbers. The dependent variable is usually the response time (RT) to reach a decision (e.g., which of these two symbols or numbers is the larger).

In such experiments, RTs have been found to decrease as the numerical separation between numbers (or the split between the ordered symbols) increases. Such a pattern is referred to as the distance effect. A different effect is that RTs for pairs of digits separated by equal distances are not always equal. For instance, for stimuli formed by

We thank M. Lorez Arnaiz for her comments in the early stages of this manuscript and S. Solla for her encouragement and comments on the initial manuscript. Correspondence concerning this article should be addressed to A. Saal, Roca 850, 1663 San Miguel, Buenos Aires, Argentina (e-mail: asaal@ungs.edu.ar). pairs of large digits (e.g., 8 and 9) the RT is relatively shorter for the instruction "select the larger" than for the instruction "select the smaller," and the reverse is true for pairs of smaller digits, such as 1 and 2. This is so, presumably, because each task induces a congruent "natural" reference and the RT is shorter when the stimulus pair is closer to the congruent reference. This effect is referred to as the semantic congruity effect (Banks et al., 1976; Jamieson \& Petrusic, 1975). Finally, investigators often find end effects in some of these experiments (Banks, 1977; Leth-Steensen \& Marley, 2000; Moyer \& Dumais, 1978). For example, the RT is shorter if one of the stimuli in the trial pair is an endpoint of the stimulus range.

Welford (1960) proposed to model the RT in a variety of comparison experiments, essentially as a logarithmic function of the split or separation between the compared stimuli:

$$
\mathrm{RT}=a \log \left[\frac{\text { larger stimulus }}{\text { split }}\right]+b .
$$

Equation 1 captures the essence of the distance effect as a peak (with a logarithmic profile) in the RT-versus-split plot.

In number comparisons, the congruity effect was first observed in selection tasks between digits (Banks et al., 1976). This effect was also found in two-digit number classification tasks (Dehaene, 1989), and more elaborate expressions were proposed (Dehaene, 1989) to describe the distance and the congruity effects (see Equations 2-5). In these new expressions, the former effect remains a split effect; for the latter effect, two reference points (near the extremes of the stimulus range) are explicitly introduced in the corresponding RT equations. 
In contrast with these heuristic formulae, other models describe decision experiments as a dynamical process. Time is usually introduced by counting the cycles needed to accumulate stochastic information up to a critical level (Laming, 1968; Stone, 1960; more recently, Leth-Steensen \& Marley, 2000; Link, 1990; Ratcliff, Van Zandt, \& McKoon, 1999; Usher \& McClelland, 2001).

The aim of this paper is to introduce a theoretical account for Dehaene's (1989) RT formulae. The outcome of the theoretical construction is an evidence accrual model (Petrusic, 1992) for the variety of point view, developed initially by Jamieson and Petrusic (1975) and explored more fully by Holyoak (1978), Dehaene (1989), and Petrusic (1992). More specific to our recursive model are the exponential accumulation rate and the accumulation stopping conditions. These features are directly associated with the distance effect and the congruity effect. The end effect results neither from the dynamical behavior nor from the stopping conditions, but it will be accounted for by a plausible selection of the encoded stimuli. We will discuss differences and similarities with other models and compare the predictions of the recursive model with available data from selection and classification tasks. Furthermore, we will discuss incorrect answers and compare model simulations with data from the observed interparticipant dispersion of the mean RT.

\section{The Recursive Model}

The interpretation of the congruity effect in terms of reference points was carefully discussed soon after it was found in digit comparisons. The general conclusions strengthen previous ideas developed in relation to symbolic magnitude comparisons and evidence accrual processes. In Holyoak's (1978) words, "Such comparisons involve an analog process (Moyer \& Landauer, 1967) that may be simulated with random walk models (Buckley \& Gillman, 1974), that the task question has an effect on the comparison process (Banks et al., 1976), that this effect results from a change of the reference point (Marks, 1972), and that the distances from a reference point may be evaluated as a subjective ratio (Jamieson \& Petrusic, 1975)" (p. 236). Here, we propose a computational connectionist model based on a specific accrual dynamic that fulfills each of these requirements.

The underlying accrual process. The following heuristic expressions describe the distance effect and the congruity effect for both classification and selection experiments. These expressions result (Dehaene, 1989) from an extension of the discriminability model originally proposed for selection experiments not related to number comparisons (Jamieson \& Petrusic, 1975):

$$
\begin{aligned}
& \mathrm{RT}[\text { classify, } \operatorname{trg}>\mathrm{std}]=a \log \left[\frac{\left(\mathrm{ref}_{\max }\right)-(\mathrm{std})}{(\operatorname{trg})-(\mathrm{std})}\right]+b, \\
& \mathrm{RT}[\text { classify, } \operatorname{trg}<\mathrm{std}]=a \log \left[\frac{\left(\mathrm{ref}_{\text {min }}\right)-(\mathrm{std})}{(\operatorname{trg})-(\mathrm{std})}\right]+b,
\end{aligned}
$$

$$
\begin{gathered}
\mathrm{RT}[\text { select }- \text { larger }]=a \log \left[\frac{\left(\mathrm{ref}_{\max }\right)-(\mathrm{S})}{(\mathrm{L})-(\mathrm{S})}\right]+b, \\
\mathrm{RT}[\text { select }- \text { smaller }]=a \log \left[\frac{\left(\mathrm{ref}_{\min }\right)-(\mathrm{L})}{(\mathrm{S})-(\mathrm{L})}\right]+b .
\end{gathered}
$$

The abbreviations std and $\operatorname{trg}$ are used for standard and target, respectively. $\mathrm{L}$ is the larger and $\mathrm{S}$ is the smaller of the numbers to be compared. Notice the parenthetical notation: $(x)$ is the model's numerical representation or code for stimulus $x$. The code values ( $\left.\mathrm{ref}_{\max }\right)$ and $\left(\mathrm{ref}_{\min }\right)$ are related to the extremes of the number interval.

A clue to understanding the underlying dynamics involved in a decision task may be provided by the logarithmic dependence observed in Equations 1-5. It suggests that a decision is the outcome of a relatively simple recurrent process. In fact, any recurrent mechanism whose final effect is to change (at a constant rate) an effective neural activity $A$ until it reaches a threshold value of $A_{\text {th }}$ will automatically generate an RT with a logarithmic time dependence.

Figure 1 illustrates the recurrent mechanism using a perceptron unit with gain parameter $\lambda$ as a schematic model. One of the inputs is a continuously available signal $\mu d$ arriving from a different structure. The other input is a feedback signal that sets up the recurrent process. Therefore, after $k$ cycles the perceptron's output $A_{k}$ is the result of the combined input signals $A_{k-1}$ (from the previous cycle output) and $\mu d$.

While incoming signals are small, output is proportional to input (nonlinear effects will not be discussed in this first approach) and

$$
A_{k}=\lambda\left(A_{k-1}+\mu d\right) \text {. }
$$

This linear recurrence equation can be explicitly solved by inspection. We see that $A$ evolves from $A_{0}=0$ to an

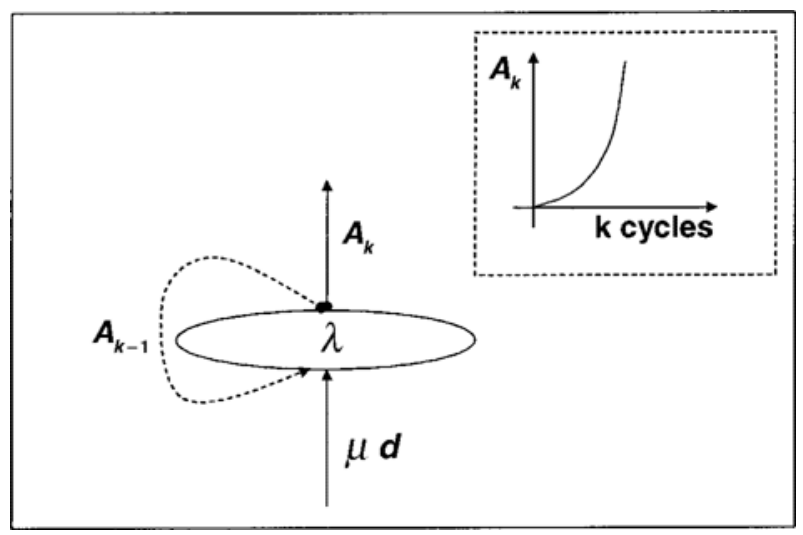

Figure 1. The recurrent accrual process. The incoming signal $\mu d$ and the feedback signal set up the recurrent process. $\lambda$ is the linear perceptron's gain parameter. The insert shows the exponential growth of the accrual. 
accrual $A_{k}=d \lambda \mu\left(1+\lambda+\lambda^{2}+\ldots+\lambda^{k-1}\right)$, after $k$ cycles. The sum of this finite geometrical series grows exponentially with the number of cycles. A simple rearrangement of terms leads to

$$
A_{k}=d \frac{\lambda^{k}-1}{q}, \text { where } q=\frac{\lambda-1}{\mu \lambda} .
$$

The accrual may be positive or negative depending on the sign of $d$. If the process stops when $A_{k}$ reaches the threshold $A_{t h}$, the total number of demanded cycles is obtained by solving Equation 7 for $k$ when $A_{k} \approx A_{t h}$ :

$$
k=\frac{1}{\log \lambda} \log \left(q \frac{A_{t h}+d}{d}+1-q\right) .
$$

The recurrent perceptron example illustrates how the mean time needed for the accrual to reach a threshold depends logarithmically on the ratio between the threshold value itself and on $d$. In Equations 1-5, the distance effect is modeled with similar scaled expressions. Therefore, it seems natural to associate $d$ with the split or numerical distance between the encoded stimuli. This leads us to propose

$$
d=(n)-(m) .
$$

Here, $n$ and $m$ are the two compared stimuli. This interpretation of $d$ is more transparent if $q=1$. The parameter $q$ has no counterpart in the discriminability model; therefore, to proceed further, we set $q$ at 1 . This constraint will be released later.

The stopping conditions and the congruity effect. In this recursive model, the exponential accrual and Equation 9 are at the core of the distance effect with a logarithmic profile. To account for the congruity effect, we will choose an explicit accrual threshold for each task and stimulus pair. The guide to determining which accrual threshold is the correct one will be a match between the model and Equations 2-5 when $q=1$.

As an example, we will first consider the select-larger experiments. In selection tasks, the stimuli are usually presented simultaneously in left and right panels on the monitor screen. We will conventionally call $m$ and $n$ the stimuli in the left and right panels, respectively. A direct comparison between Equations 8 and 4 shows that the RT from both models may be equal (recall that $q=1$ ) if

$$
\frac{\left(\mathrm{ref}_{\max }\right)-(\mathrm{S})}{(\mathrm{L})-(\mathrm{S})}=\frac{A_{t h}+d}{d}=\frac{A_{t h}+(n)-(m)}{(n)-(m)} .
$$

Rearranging this equation, we get $(m)-A_{t h}=$ $\left(\operatorname{ref}_{\max }\right)$ when $m>n$, and $(n)+A_{t h}=\left(\operatorname{ref}_{\max }\right)$ when $n>$ $m$. From this analysis, two different thresholds seem to be necessary. Nevertheless, the proposed accumulation is only one, and Equation 10 may be interpreted as the result of an accrual that continues until the first of the two following stopping conditions is satisfied:

$$
(m)-A_{k} \geq\left(\operatorname{ref}_{\max }\right)
$$

and

$$
(n)+A_{k} \geq\left(\operatorname{ref}_{\max }\right)
$$

In both of Equations 11, the left side grows exponentially until it reaches $\left(\mathrm{ref}_{\max }\right)$ from below and then stops (recall, $-A_{k}>0$ if $m>n$ ).

These equations may be illustrated graphically. In Figure $2 \mathrm{~A}$, the accrual from the recurrent process feeds forward two units. The plus and minus signs serve as reminders of the appropriate addition of signals. One of these units will fire a response signal when the stopping condition is satisfied. A positive accrual will activate the right unit, and a negative accrual will activate the left unit. Since the sign of the accrual is determined by the sign of $d \equiv$ $(n)-(m)$ (see Equation 7), the unit on the right will fire if $(n)>(m)$ and the unit on the left will fire if $(m)>(n)$. These are the correct answers expected for a select-larger task. Incorrect answers will later be considered as related to deviations from this deterministic approach.

The same analysis applied to the select-smaller experiments leads to $(m)-A_{t h}=\left(\operatorname{ref}_{\min }\right)$ for $m<n$, and to $(n)+$ $A_{t h}=\left(\mathrm{ref}_{\min }\right)$ for $n<m$. The respective stopping conditions are

$$
(m)-A_{k} \leq\left(\text { ref }_{\text {min }}\right)
$$

and

$$
(n)+A_{k} \leq\left(\operatorname{ref}_{\min }\right) .
$$

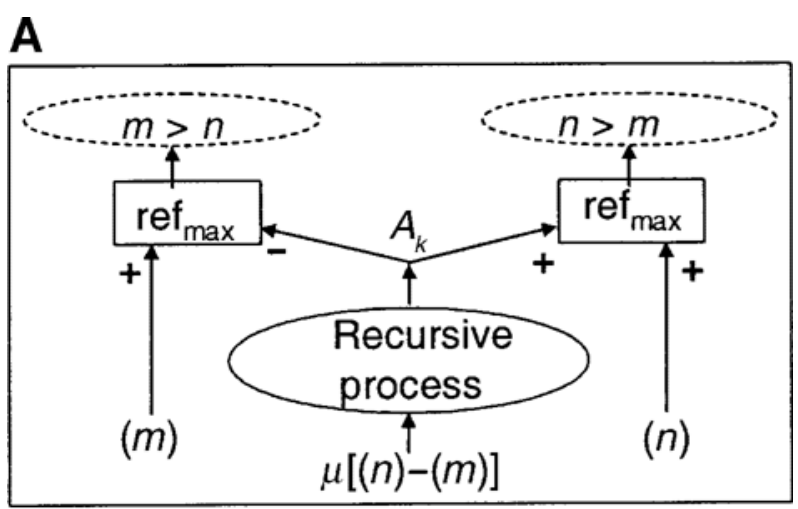

B

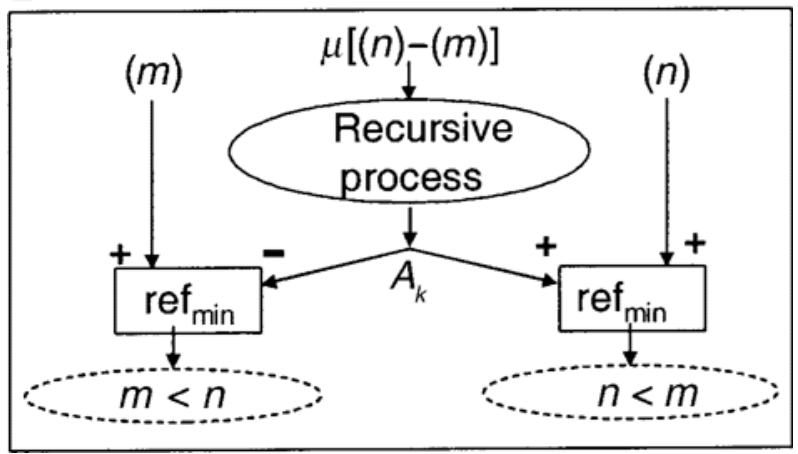

Figure 2. Schematic representation of the recursive dynamics in a select-larger (A) and a select-shorter (B) experiment. For both tasks, the accrual $A_{k}$ will either activate the left or the right output unit, depending on the sign of the input $d=(n)-(m)$. For each task, RT is controlled by the recursive gain, the signal strength $\mu$, and the reference parameter. 
Unlike in the former stopping conditions, $\left(\mathrm{ref}_{\min }\right)$ is reached from above.

Notice that the dynamical process implied by Equations 11 and 12 may be reinterpreted in terms of two (alternative) dynamical variables: $(n)_{k} \equiv(n)+A_{k}$ and $(m)_{k} \equiv$ ( $m)-A_{k}$. A description based on these variables gives rise to an alternative mathematical description based on independent accumulators. Usher and McClelland (2001) have recently developed a similar approach to study perceptual comparisons. In this approach, the two accumulators have inhibitory interconnections.

Figure 2B illustrates the stopping conditions for the select-smaller task. The most significant difference between the selection tasks lies in the use of different references.

The classification task may be analyzed in a similar way. Comparing Equations 2 and 3 with Equation 8, we get $A_{t h}+(\operatorname{trg})=\left(\mathrm{ref}_{\max }\right)$ and $A_{t h}+(\operatorname{trg})=\left(\mathrm{ref}_{\min }\right)$ for (trg) $>$ (std) and (std) $>$ (trg), respectively. The classification stopping conditions involve only one of the stimuli (not both of them, as in each selection task) and both references (not one, as in each selection task).

Deterministic RT equations. The accrual threshold has now been expressed in terms of the two references and the stimuli. To derive the model RT equations, we replace these prescriptions in Equation 8. Therefore, for any value of $q$ we find (see Equations 13-16 at the bottom of this page).

Here, we have used RT $=\tau k+b$, where $\tau$ and $b$ denote the mean time per cycle and the mean residual nondecisional time, respectively. These RT equations become identical to those of the discriminability model in the limit $q=1$.

Behind these mathematical expressions lie essentially two phenomenological inputs from RT experiments: distance effect and semantic congruity effect. Congruity effect is associated with reference values, whereas distance effect is related to the $1 /[(n)-(m)]$ factor. In the Experimental Validation section, we will show that the end effect is masked in the encoded stimuli.

On the other hand, the embracing functional (i.e., the $\log$ function) and the $q$ coefficient result from the peculiarities of the accrual mechanism. This mechanism, in- herited from the discriminability model, accumulates at an exponential rate.

Nevertheless, in the limit when $\lambda=1$, the accrual is merely additive and the exponential accrual becomes a linear function of the number of recursive cycles:

$$
A_{k}=d \lambda \mu\left(1+\lambda+\lambda^{2}+\ldots+\lambda^{k-1}\right) \stackrel{\lambda \rightarrow 1}{\rightarrow} d \mu k .
$$

Encoded stimuli. To complete the model description, we assume that the internal numerical representation of the stimuli is related to the way in which these stimuli were learned (Leth-Steensen \& Marley, 2000; Trabasso \& Riley, 1975; Trabasso et al., 1975). The learning process associated with the variety of stimuli we discuss here is too complex for the current stage of development of the recursive model. Therefore, in a first heuristic approach, we consider the code function (i.e., the stimuluscode relationship) as a free set of values to be fitted from empirical data (see the Discussion at the end of the Experimental Validation section). In this framework, the end effect follows from endpoint codes that are relatively spaced out from the others.

Since the ratio $[($ reference $)-(\ldots)] /[(\ldots)-(\ldots)]$ is invariant under any linear transformation of the codes, we are free to scale the interval of the encoded stimuli at will. Hence, with no loss of generality, we choose the interval extremes to be $\left(n_{\min }\right),\left(n_{\max }\right)=0,1$. Furthermore, we introduce

$$
\begin{aligned}
& \left(\operatorname{ref}_{\max }\right)=\left(n_{\max }\right)+\Theta_{\max }=1+\Theta_{\max }, \\
& \left(\operatorname{ref}_{\min }\right)=\left(n_{\min }\right)+\Theta_{\text {min }}=0-\Theta_{\text {min }} .
\end{aligned}
$$

There is only a formal difference between the references and the $\Theta$ values. Nevertheless, in the network representation $\Theta$ will become a bias parameter interpretable as a stimulus-independent external signal.

The network representation. Here, we will use a neural network description to develop a graphical representation of the recursive model. This approach may help to identify the model's pros and cons and to suggest further developments. It may also be more transparent for computational purposes when noisy signals are considered. In this context, we assume that the perceptrons are not to be interpreted as single neurons, but, more

$$
\begin{aligned}
& \mathrm{RT}[\text { classify, } \operatorname{trg}>\mathrm{std}]=a \log \left[q \frac{\left(\mathrm{ref}_{\max }\right)-(\mathrm{std})}{(\operatorname{trg})-(\mathrm{std})}+1-q\right]+b, \\
& \mathrm{RT}[\text { classify, } \operatorname{trg}<\mathrm{std}]=a \log \left[q \frac{\left(\mathrm{ref}_{\min }\right)-(\mathrm{std})}{(\operatorname{trg})-(\mathrm{std})}+1-q\right]+b, \\
& \mathrm{RT}[\text { select }-\operatorname{larger}]=a \log \left[q \frac{\left(\operatorname{ref}_{\max }\right)-\operatorname{Min}[(\mathrm{m}),(\mathrm{n})]}{|(n)-(m)|}+1-q\right]+b, \\
& \mathrm{RT}[\text { select }- \text { smaller }]=a \log \left[q \frac{\left(\operatorname{ref}_{\min }\right)-\operatorname{Max}[(\mathrm{m}),(\mathrm{n})]}{|(n)-(m)|}+1-q\right]+b .
\end{aligned}
$$




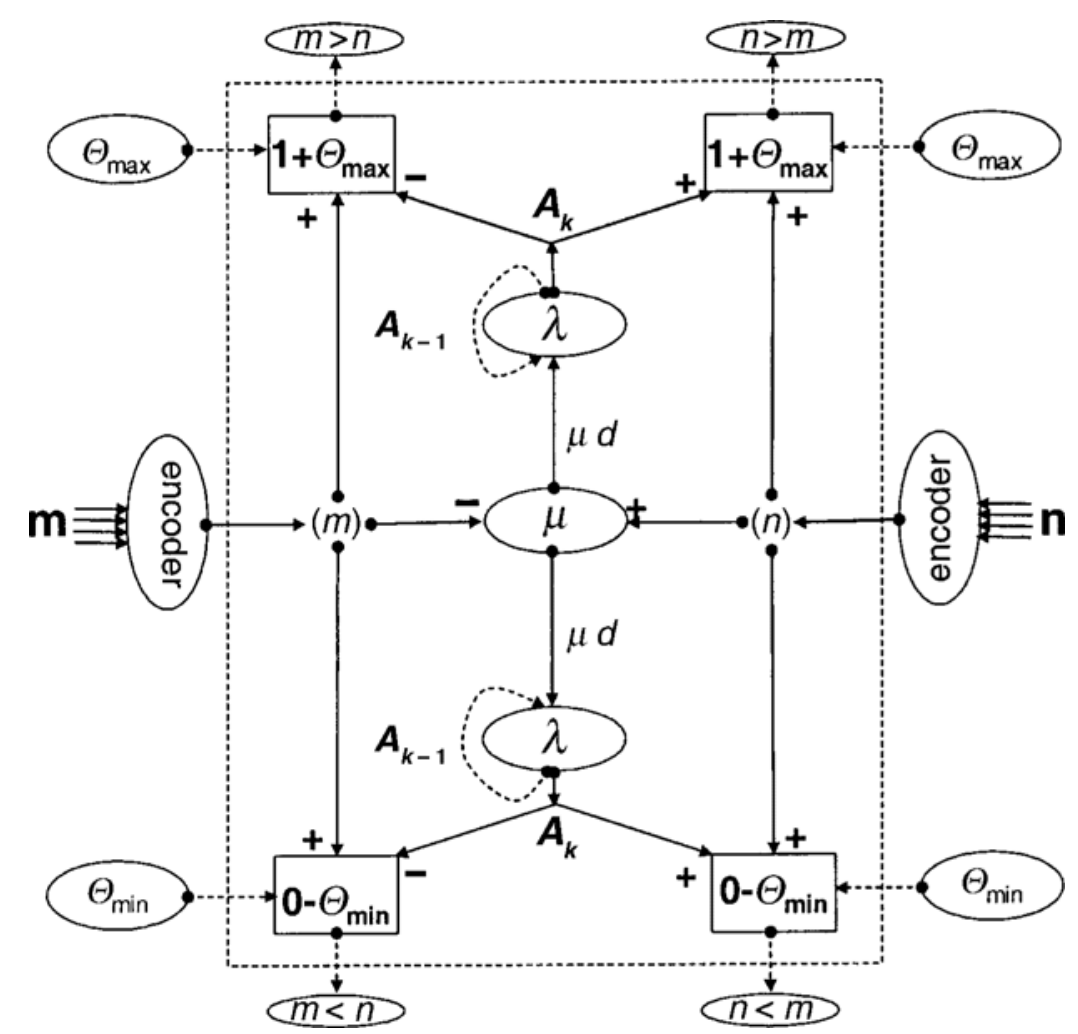

Figure 3. Schematic network for a selection experiment. The dashed rectangle limits the decision processes. External to it are the needed inputs and the expected outputs. Within the dashed rectangle, oval units are the recurrent perceptrons and the difference calculator with gain factors $\lambda$ and $\mu$, respectively. The labels in the rectangular units indicate the bias of the output perceptrons.

likely, as pools of neurons that behave collectively as effective neural structures with emerging properties.

Figure 3 illustrates the selection network. It behaves in a similar way for each of its four outputs. Output units are modeled roughly as on-off perceptrons. Any of them receives two inputs from different sources: the accrual and one of the encoded stimuli. At first, the encoded stimulus by itself is not strong enough to fire the on-off perceptron, but after $k$ recurrent cycles, the added accrual contribution sets the output unit to signal for the motor answer. The recurrent perceptron is fed by the linear neuron (with gain $\mu$ ), which computes the $(n)-(m)$ difference. The inputs to this difference-calculating neuron are the encoded stimuli. The output from each encoder unit also feeds the corresponding on-off output units.

The biases of the output neurons are $1+\Theta_{\max }$ and $0-\Theta_{\min }$ (see Equations 17 and 18). Notice the terms added to the $\Theta$ bias $\left(n_{\max }=1\right.$ and $\left.n_{\min }=0\right)$. These terms can be considered as fixed inputs to the on-off perceptrons. Thus, they introduce explicit information from the extremes of the stimulus range. On the other hand, the $\Theta$ bias modulates the network behavior and adjusts it to the actual task. We may imagine a scenario with naturally high $\Theta$ values. Lowering one particular threshold allows the corresponding output perceptron to become active while the other remains inhibited. A low value of $\Theta_{\max }$ and of $\Theta_{\min }$ is expected for the select-larger and the select-smaller tasks.

Figure 4 illustrates the classification network. Again, the building blocks are the recurrent accrual process (see Figure 1 or Equation 6) and the stopping conditions. The correct stopping conditions for the classification task include two output neurons with different biases. During a selection task, only one $\Theta$ value is low. During the classification task, in contrast, the two references are significant (Dehaene, 1989). Therefore, both $\Theta$ values are low.

Beyond the deterministic approach. The recursive model was explicitly constructed, by combining noiseless signal accrual and stopping conditions to provide a theoretical support for a successful set of empirical formulae. The model may be extended to incorporate noisy signals and parameter fluctuations. In the Experimental Validation section, we will discuss incorrect answers in terms of stochastic fluctuations from the codes mean val- 


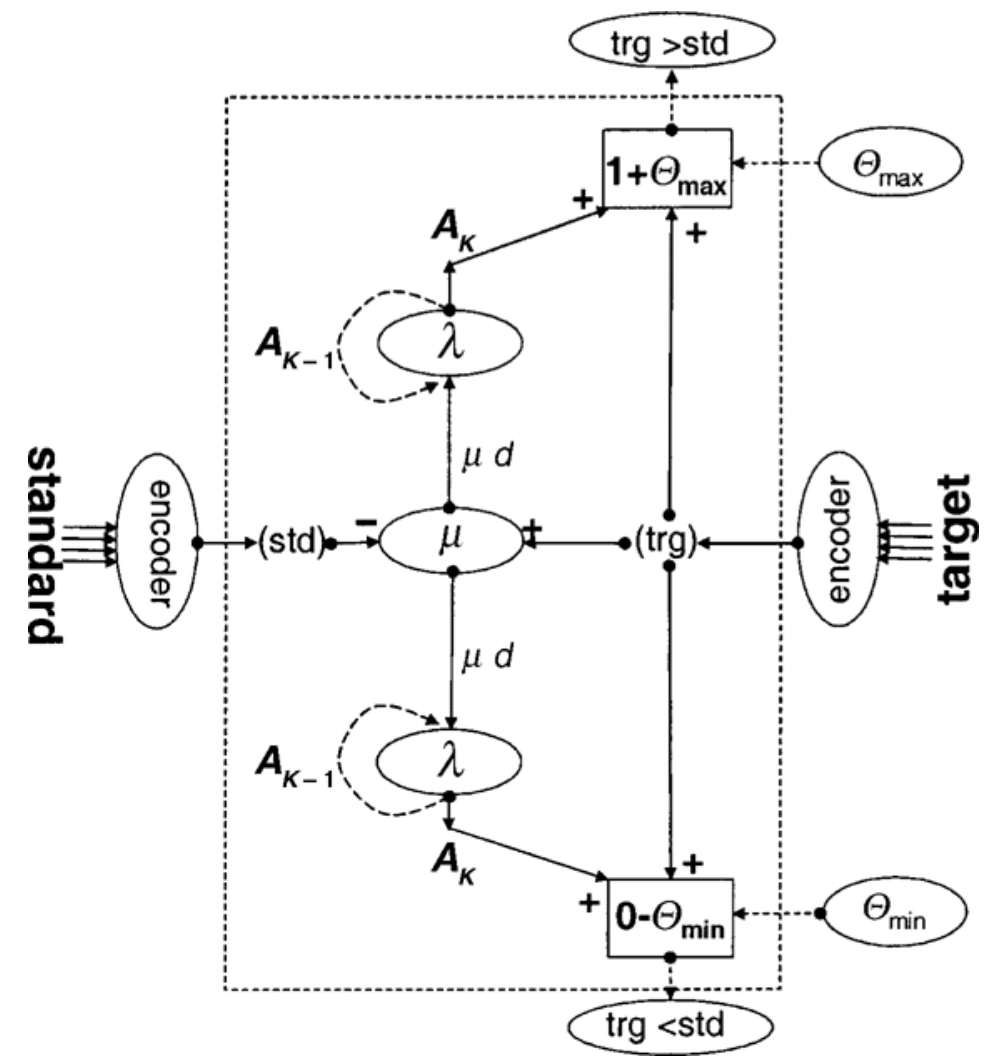

Figure 4. Schematic network for a classification task. The dashed rectangle limits the decision processes. External to it are the needed inputs and the expected outputs. Within the dashed rectangle, oval units are the recurrent perceptrons and the difference calculator with gain factors $\lambda$ and $\mu$, respectively. The abbreviations trg and std stand for target and standard, respectively.

ues. Furthermore, we will show that the variance of the mean RT across participants may be attributed to variations of the recursive gain parameter among individuals.

\section{Comparison With Other Models}

The recursive model deals with the three major RT effects (distance, end, and semantic congruity) in a way that is partially different from that followed in other evidence accrual models. In this section, we will focus on these differences and similarities in an attempt to differentiate the recursive model structure from other dynamical approaches.

Random walk models. In the classical random walk and related diffusion models, the noisy information from a stimulus is additively accumulated over time toward one of two decision boundaries. These models accumulate information sampled at discrete time steps (random walk) or continuously (diffusion). Depending on the sign of the mean rate of accumulation, the accrual evolves to one boundary or the other; if the process arrives (statistically) at the incorrect boundary, it is interpreted as an incorrect answer.

In the recursive model, incorrect answers are interpreted in a similar way. When noisy signals associated with stochastic variations of the codes and the parameters are taken into consideration, the recursive process bears a resemblance to the random walk model. The most noticeable difference between random walk models and the recursive model is the nature of the accumulator. The former models generally use an additive accrual process, whereas the recursive accrual may have an exponential accumulation rate (i.e., $\lambda>1$ ). In the recursive model, higher values of $\lambda$ increase the response speed, but they also favor more incorrect answers. This monotonous relationship between speed and accuracy may be intuitively understood: In a classical random walk, equally weighted information is accumulated at each evidence accrual event, whereas in the recursive model, after $k$ cycles the stochastic contribution of the first event is weighted with $\lambda^{k}$, the second with $\lambda^{k-1}$, and so on. Thus, the first cycles are the most relevant to determining the final boundary. Therefore, higher $\lambda$ values increase the number of incorrect answers.

In random walk models, the congruity effect is usually achieved (Ashby, 1983; Link \& Heath, 1975) by biasing the starting point. In the recursive model, congruity is built in the barrier (i.e., the accrual thresholds) and depends explicitly on the task and the stimuli. For 
example, in a classification experiment the accrual thresholds are $\left(\mathrm{ref}_{\max }\right)-(\mathrm{std})$ and $\left(\mathrm{ref}_{\min }\right)-(\mathrm{std})$. This role of the standard code resembles the congruity bias in random walk models.

Random walk models are frequently used in relation to perceptual comparisons between confusable stimuli. Recently, Usher and McClelland (2001) developed the leaky, competing accumulator model to describe comparisons in terms of different accruals that evolve under constraints from three simultaneous mechanisms: competing accumulation, recurrent feedback, and decay or leak of accumulation. The authors solve the general model for a binary perceptual choice and test it in RT and timecontrolled experiments. For binary choices, the model reduces to an evolution equation analogous to Equation 6 . Inspecting both equations, we find that $\lambda$ in the recursive model is similar to the sum of parameters from the leaky, the competing, and the recurrent-feedback mechanisms. Also, the term $\lambda \mu d$ with the stimuli information presented in Equation 6 has a similar counterpart (related to the numerical representation of the stimuli) in the leaky model equation. The most noticeable difference is that in the leaky model the accrual threshold is independent of the stimuli and, therefore, no congruity effect is described.

The diffusion model is an important extension of random walk models. It has been repeatedly tested with perceptual two-choice comparison data from different experiments (Ratcliff, 1978, 1988; Ratcliff \& Rouder, 1998; Ratcliff et al., 1999). Ratcliff (1978) introduced the assumption that there may be variability in the mean direction of drift from trial to trial within a single experimental condition. This presumably accounts for variability in memory strength for individual stimuli. Such variance closely controls the rate of incorrect answers. This memory signal is not present in the recursive model. Nevertheless, the speed parameter $\lambda$ also modulates the rate of incorrect answers, but through a different mechanism.

Symbolic comparison model. Experiments with linear syllogistic learning tasks (Leth-Steensen \& Marley, 2000; Trabasso et al., 1975) use a set of six discriminable symbols and assign an artificial order relation to them. During the first experimental stage, participants learn the order relation between pairs of adjacent symbols. Learning progresses through trial-and-error essays, with the help of computer feedback. Next, during the test stage, the participants are asked to decide the order relation between any two stimuli (adjacent or not). The test phase is a selection experiment randomly set to a select-larger or a select-smaller task. To be precise, the selection in this case is between the categories taller and shorter. Within the recursive model, these are equivalent to larger and smaller. Therefore, we use the latter terms throughout the text.

Leth-Steensen and Marley (2000) developed a symbolic comparison model that describes the RT measurements from linear syllogistic learning tasks. In this computational connectionist model, two perceptrons in a hidden layer transform the vector representation of each stimulus into a code number, and an output perceptron computes the difference between these codes. After the implementation of the back propagation algorithm (to simulate the learning stage), the learned codes are almost linearly related to the relative order of the stimuli.

To simulate the experimental test stage for each stimulus pair, the corresponding codes are processed in two simultaneous and independent pathways. Each pathway feeds a different additive accumulator, and RT is determined by the accrual that first reaches a threshold value.

Both accrual mechanisms accumulate activity signals modulated by additive terms and exponential cut-offs (the exponential similarity function) related to right- or lefthand answers and task alternatives. The effective accrual is determined mainly by the actual task and correct-hand answer, but interference effects from the other task and hand are taken into consideration with modifying terms.

An important difference between the recursive model and the symbolic comparison model is the modeling of the learning stage. This is beyond the scope of the recursive model in its current stage of development, and codes are evaluated after a fitting process. It is instructive to compare the codes that result from each model, because they reflect the way each model treats the end effect.

In the recursive model, we propose that the end effect is accounted for by a singular behavior of the endpoint codes. To explain the end effect, some authors (e.g., Trabasso \& Riley, 1975; Trabasso et al., 1975) correlate the relevance of the largest and smallest stimuli during the learning phase with the corresponding short RT during the test phase. In line with these proposals, we may expect, as a rule of thumb, that the distance between codes of ordered symbols correlates to the relevance of the corresponding symbols during the learning stage. Since the linear syllogistic task-learning method favors a different attitude toward the extreme symbols, this speculative reasoning predicts a code function that discriminates between the extreme and middle codes. In the recursive model, this conjecture is well satisfied by the fitted code values (see insert in Figure 7). Notice that the middle codes are linear with the ordering of the stimuli, but the endpoint codes stretch away from the linear behavior. Thus, as expected from the $|(n)-(m)|$ denominator in Equations 15 and 16, the end-effect fast answers may be attributed to the stretching away of the endpoint codes.

In contrast to the recursive model, the symbolic comparison model explains the end effect in terms of the twopathway strategy. One of the two paths is faster when the stimulus pair includes an endpoint stimulus. Technically, this is achieved by the exponential similarity function, which modifies the effective accrual in the end-anchor pathway.

In the next section, we will show that both models provide similar fits to the same data. This fact may draw attention because of the difference between the additive and the exponential accrual growth proposed by the symbolic comparison model and the recursive model, respectively. Nevertheless, the two accumulators are additive at the limit $\lambda \rightarrow 1$; therefore, a value of $\lambda \approx 1$ could be anticipated for these data. We will provide some evi- 
dence of such a value by comparing the interparticipant dispersion from the mean RT with the recursive model prediction.

Each model deals with the congruity effect differently. In the recursive model, the effect is related to the accrual stopping conditions, which operate as a differential bias mechanism [see the $(m)$ and $(n)$ contributions to the output units in Figure 3]. In the symbolic comparison model, the effect is built in the model through specific assumptions about the role of semantic interference (Cohen, Dunbar, \& McClelland, 1990) between competing instructional pathways.

Finally, each model's description of the distance effect depends on the neural unit that computes the difference $(n)-(m)$. The output of this unit feeds the accumulation process, thus giving rise to the distance effect. Since the exponential accrual and the additive accumulation are comparable if $\lambda \approx 1$, the origin of the distance effect is similar in both models.

\section{Experimental Validation}

In this section, we will apply the recursive model to published data on three experimental paradigms. In each case, we will fit the data points and compare our results with the authors' quantitative conclusions. We will discuss the code functions used for the different data sets and, when incorrect answers are reported, we will analyze them in relation to the simulations of the recursive model.

In RT experiments, the fitting parameters of the recursive model are the slope $a$, the coefficient $q$, the residual time $b$, the task reference, and the encoded stimuli (see Equations 13-16). Since $a=\tau / \log (\lambda)$ and $q=(\lambda-$ $1) /(\lambda \mu)$, to determine $\lambda, \mu$, and $\tau$, more detailed data, such as the RT standard deviation or incorrect answers, are needed.

Two-digit number classification task. We will first consider a classification experiment (Dehaene, 1989, Experiment 2). The 5 participants compared target numbers from 20 to 99 with the standard of 75 . The experiment provides 79 data points. For each participant, the targets between 20 and 74 appeared randomly in three trials, and those between 76 and 99 appeared in 7 trials. This ensured equiprobability of "larger" and "smaller" responses. Correct RTs were averaged across subjects and trials to yield the data points shown in Figure 5.

Following Dehaene's (1989) empirical findings, we assume a linear code function. Furthermore, setting $q \equiv 1$, we trivially reproduce his results with the same number of parameters. Moreover, if the constraint $q \equiv 1$ is released and $q$ is used as an extra free parameter, we consistently find the regression value $q=0.98$, with no significant improvement (see the regression curve in Figure 5, with $R^{2}=.844$ ).

Nevertheless, we will show that in other experiments the regression is improved if $q$ is considered as a fitting parameter.

Dehaene (1989, Experiment 1) also performed a classification experiment with standards randomly chosen from among $35,45,55,65$, and 75 . The author tested the discriminability model and found $R^{2}=.54$ with essentially two free parameters $(a$ and $b)$. In both experiments, the code function was considered as linear and the references were taken near the stimuli's endpoints[i.e., $\left(\operatorname{ref}_{\max }\right)=1$, $\left(\mathrm{ref}_{\min }\right)=0$ in the recursive model]; the results were insensitive to variations around these values. The author concluded that, in spite of the low correlation coefficient,

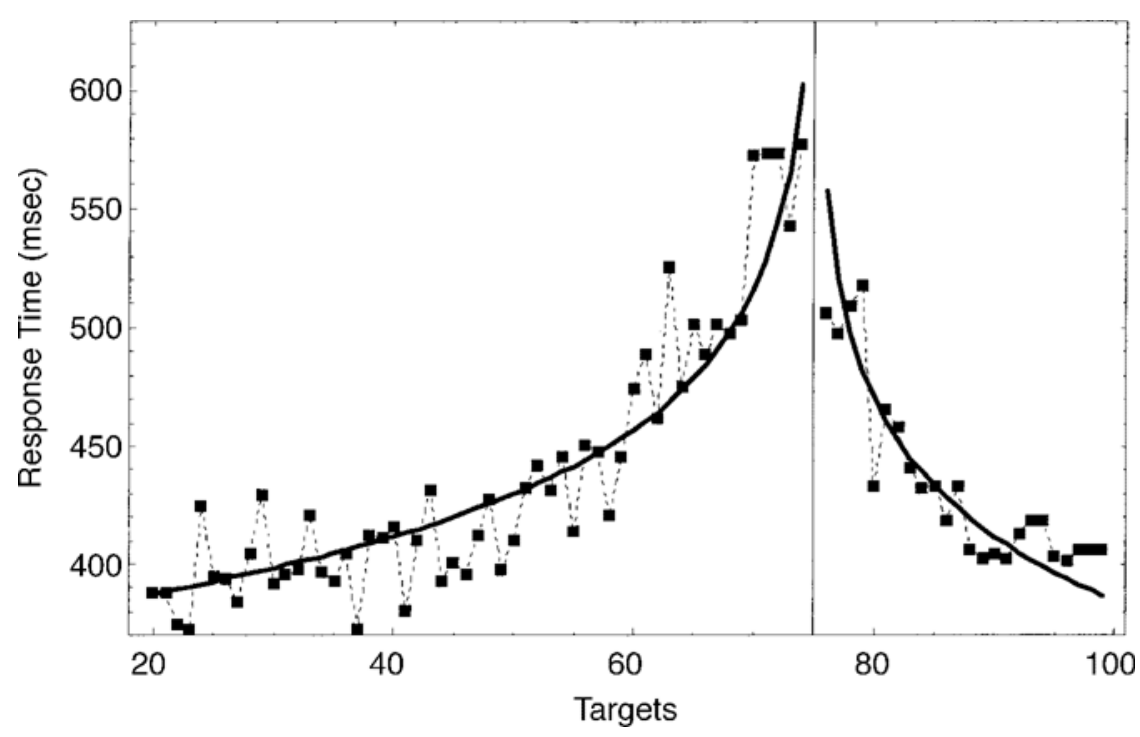

Figure 5. Data from Dehaene (1989). Response time measurements for classification comparisons between the standard number (75) and two-digit numbers between 20 and 99 (dashed line with filled squares). The solid line $\left(R^{2}=.844\right)$ is the regression curve from the recursive model. 
no further validation could be expected from the particular data, due to the rather strong statistical fluctuations observed in the 240 data points (presumably, because each data point was the average of 20 trials—only 1 per participant). In accordance with Dehaene's (1989) reasoning about the strong statistical fluctuations, enhancing the parametric space $\left[q \neq 1,\left(\operatorname{ref}_{\max }\right) \neq 1,\left(\mathrm{ref}_{\min }\right) \neq 0\right]$ accounts for only a marginally better correlation coefficient.

Comparison of digits in selection tasks. We will next consider RT measurements from Banks et al. (1976, Experiment 2). The stimuli were digits with split 1,2, or 3 . Each of the 42 data points (21 points for the selectlarger task and 21 for the select-smaller task) was obtained by averaging across 18 participants and collapsing left and right presentations; 180 trials contributed to each data point. The authors interpreted the RT measurements with their semantic encoding model on the basis of the conditional probability for the stimuli to be near one or the other reference. The model has three intrinsic parameters in addition to the parameters of the encoding function. In the quantitative analysis, different encoding functions were tested.

The encoding function with the (marginally) best result (i.e., $R^{2}=.69$ with $3+1$ parameters) was

$$
(n)_{\text {Banks }}=1-e^{-\beta n} \text {. }
$$

To make a simple comparison between the recursive and the semantic encoding models, we restricted the set of parameters imposing $\Theta_{\max }=\Theta_{\min }=0$. This leaves four parameters free (i.e., $a, b, q, \beta$ ). We found that the recursive model has a similar performance with $R^{2}=$
.70. When the restriction on the $\Theta$ bias is released, the fit improves and $R^{2}=.84$.

Figure 6 displays the experimental and predicted RTs in an isosplit representation. For each split, there are two alternative predictions (solid lines) based on different test code functions. Treating the codes and the references as if they were additional parameters to be fitted (solid line with filled triangles) resulted in a better fit, with $R^{2}=.93$. In this case, there were 13 free parameters.

The insert in Figure 6 illustrates both code functions. The isolated triangles result from the code-to-code fit. The continuous line represents Equation 19, with $\beta=$ 0.23 . The two code functions look similar to the eye, but they represent independent fits of different test code functions. The small differences are notoriously enhanced in the RT predictions (especially the Split 1 data). The smooth code function allows the recursive model to interpolate the experimental RT trend, but it is only when the code-to-code fitted values are introduced that the predicted RT follows the details of the measured RT.

The linear syllogistic reasoning task. In this approach to symbolic comparisons (Leth-Steensen \& Marley, 2000), a collection of 30 data points were evaluated (Splits 1-5). Each of the 30 data points (15 for the selectlarger task and 15 for the select-smaller task) was obtained by averaging across 16 participants and collapsing left and right presentations; 160 trials contributed to each data point.

Figure 7 shows the RT measurements (dashed lines with filled dots) reported by Leth-Steensen and Marley (2000) in an isosplit plot. The error bars represent the

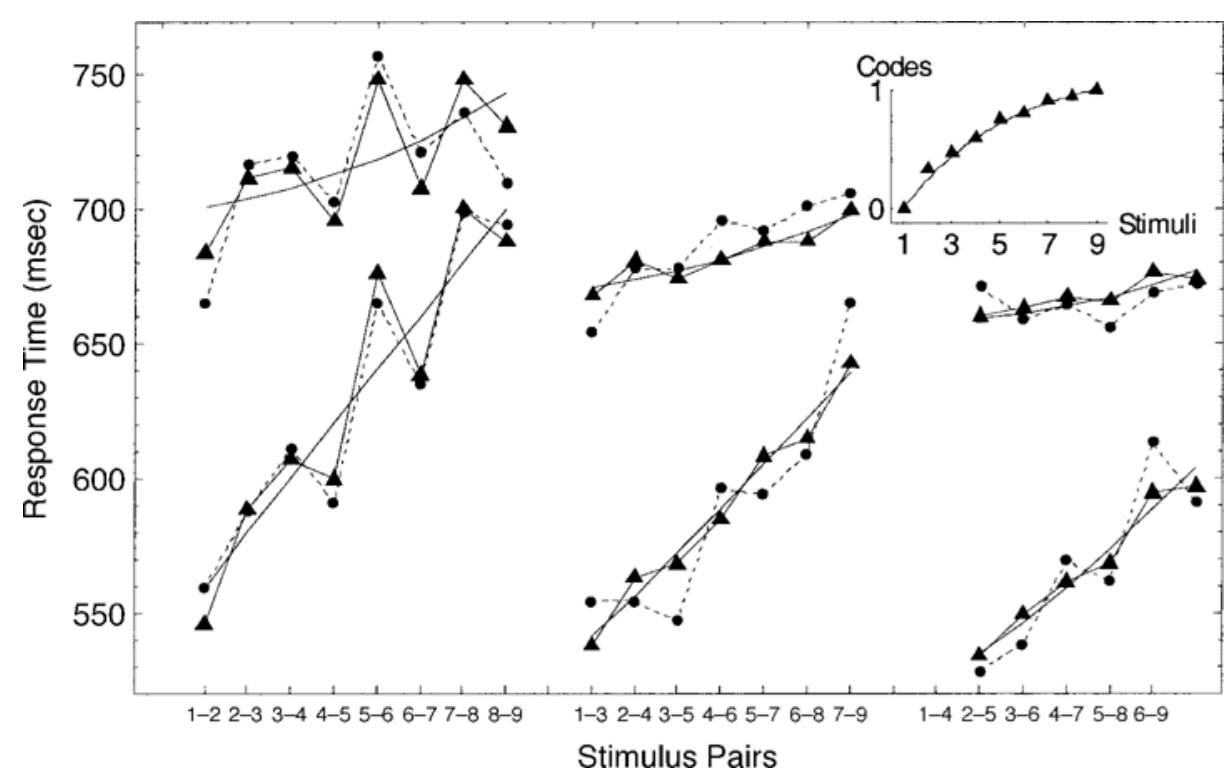

Figure 6. Data from Banks et al.'s (1976) selection measurements (dashed lines with filled circles). From left to right, the regression curves are the predictions for Splits 1, 2 and 3, respectively. For each split, the upper curves correspond to data from the select-larger task. For the sake of illustration, the RTs for select-larger data are artificially increased by $120 \mathrm{msec}$. The lower curves correspond to data from the select-shorter task. Two alternative (independent) code functions were used: The unmarked solid line corresponds to codes as in Equation 19, and the solid line with filled triangles corresponds to codes fitted as parameters. The insert illustrates the two code functions. 


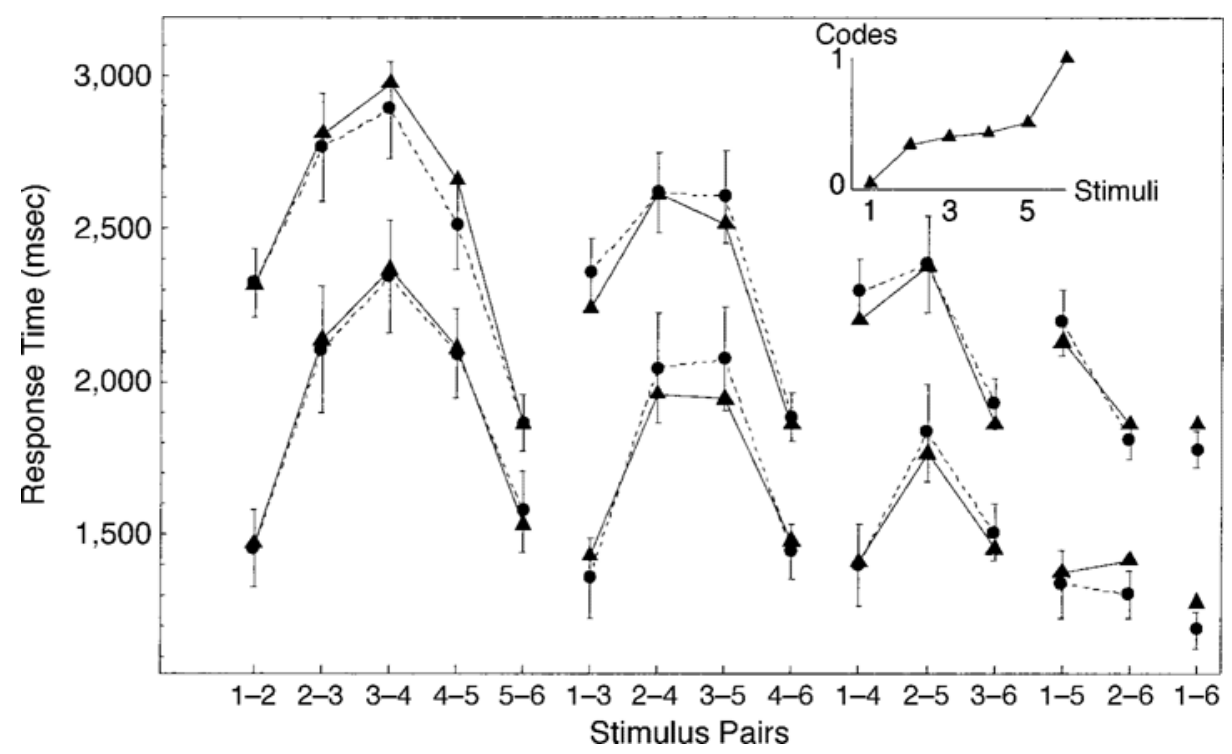

Figure 7. Data from Leth-Steensen and Marley's (2000) measurements (dashed lines with filled circles). From left to right, the regression curves (solid lines with filled triangles) represent predictions for Splits 1-5, respectively. The lower curves are for the select-shorter task. For the sake of illustration, the RTs for select-larger data are artificially increased by $\mathbf{7 0 0}$ msec. The fitted stimulus codes are shown in the insert.

$S D$ s from the mean RT across participants. The figure also displays the predictions of the recursive model (solid line with filled triangles).

The end effect is clearly present with its characteristic inverted U shape. As was discussed in the previous section, the recursive model's interpretation of this effect is related to the nonlinear code function shown in the insert. To find the regression curve from the recursive model, nine parameters were used, and we found $R^{2}=$ .969. The fitted parameters are as follows:

$$
(a, b, q)=(309.8,939.7,3.33),
$$

((1), (2), (3), (4), (5), (6)) $=(0,0.339,0.403$,

$$
0.437,0.520,1) \text {, }
$$

and

$$
\left(\Theta_{\min }, \Theta_{\max }\right)=(0.4524,0.1773) .
$$

Notice that the codes for the endpoint stimuli are set to 0 and 1 and are not free parameters. The symbolic comparison model also introduces nine fitted parameters and obtains almost the same correlation, $R^{2}=.968$ (Leth-Steensen \& Marley, 2000).

Incorrect answers. In accrual models, it is a standard practice to introduce noise sources in order to model data dispersion and incorrect answers. If, in some cycle, the encoded stimuli were statistically inverted from their natural order, the accrual would accumulate in the wrong direction and favor an incorrect answer. In Leth-Steensen and Marley's (2000) experiment, accuracy is near ceiling and the number of incorrect answers is not statistically significant. Hence, simulations only confront the qualitative behavior of the model.
To simulate incorrect answers, we added to the encoded stimuli a centered Gaussian random noise with $S D$ $\sigma_{c}=0.1$ in each recursive cycle. Due to the step shape of the test code function (see the insert in Figure 6), this error mechanism affects primarily the comparison of middle split 1 stimuli. Statistical fluctuations in codes from pairs containing endpoints or with a larger split will only rarely invert their natural order. We have estimated a correct proportion of errors (5\%-6\%) for middle split 1 data. Other stimulus pairs have a smaller proportion of observed errors $(2 \%-3 \%)$, and the corresponding simulation falls to zero. Such a qualitative behavior was also found in an attempt to simulate errors with the symbolic comparison model (this can also be seen in Leth-Steensen \& Marley, 2000, Figure 3).

The interparticipant dispersion of the mean RT. Figure 8 shows $\sigma_{<\mathrm{RT}>}$, the $S D$ from the mean RT across participants, for each stimulus pair in Leth-Steensen and Marley's (2000) experiment (dashed lines with filled circles). It is striking to notice that $\sigma_{<\mathrm{RT}>}$ reproduces the inverted U shape observed in the RT (see Figure 7). We simulated the performance of different participants (solid line with filled triangles), assigning to each of them a different set of the model parameters. ${ }^{1}$ Each calculated point (filled triangle) is a simulation of 500 different participants, each with 200 trials. The variance of most parameters does not have any significant contribution to $\sigma_{<\text {RT }>\text {. Even }}$ considering signal-to-noise ratios $<1$, the $\lambda$ variation is the only contribution that generates theoretical dispersions of the order of the empirical $\sigma_{<\mathrm{RT}>}$.

We took the parameter $\lambda-1$ from a gamma distribution with mean 0.07 and variance $0.072 / 5$. Therefore, the 


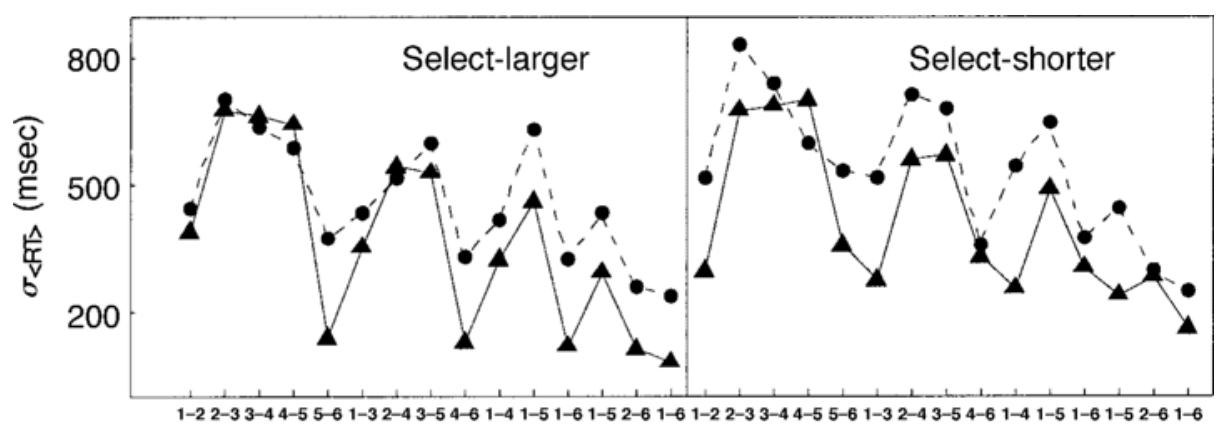

Stimulus Pairs

Figure 8. A semiquantitative comparison between the measurements from Leth-Steensen and Marley (2000) and the recursive model prediction: the empirical data measurements (dashed lines with filled circles) and model simulation of the interparticipant $S D$ of the mean RT for selection tasks (solid lines with filled triangles).

mean value of the effective gain parameter is $\lambda=1.07$. The rest of the model's parameters may be estimated using the values from Equation 20. We get $\mu=0.02$ and $\tau=21 \mathrm{msec}$. The comparison presented in Figure 8 is only semiquantitative. Further refinements would not provide new meaningful information, since the 16 participants in the comparison experiment were too few for a reliable estimation of $\sigma_{<\mathrm{RT}>}$.

It is interesting to note that the interpretation of $\sigma_{<\mathrm{RT}>}$ is very specific to the model. In fact, the recursive model's accrual evolves at an exponential rate $(\lambda>1)$, whereas other models usually propose an additive accumulation (i.e., $\lambda \equiv 1$ ) and no variation from linearity is expected.

Discussion. The code functions resulting from the fitting process were either linear as for the classification data (Figure 5), exponentially shaped as in the insert in Figure 6, or step shaped as in the insert in Figure 7. This diversity presumably originates during the learning process associated with each set of stimuli, as was stated in the proposed rule of thumb. For example, we may consider the exponential code function of Equation 19. Since, during the learning of digits, the first and best differentiated numbers are 1,2, and 3, it is the codes of these digits that remain most distinguishable from the others. Therefore, the distance between, say, (2) and (3) is larger than the distance between (3) and (4). Furthermore, in two-digit number comparisons, no number has an a priori specificity ${ }^{2}$ and the code function seems to be practically linear (Dehaene, 1989, 1990), again in accordance to the rule-of-thumb expectations. To return to the linear syllogistic reasoning task, if the rule of thumb is taken as a working hypothesis, the fitted code function makes sense. In traditional symbolic comparisons, codes are expected to be easily available, well established memory resources. In the linear syllogistic reasoning task, the new codes are developed during the learning effort of the training phase. This learning method favors a different attitude toward extreme symbols, whereas middle symbols have much less specificity. Therefore, we expect a linear growth for middle codes and two well differentiated codes for the first and sixth symbol.

\section{SUMMARY AND CONCLUSIONS}

In this article, a new accrual connectionist model for judging order relationships between two symbolic stimuli is presented. It accounts well for several important phenomena of such judgments: the distance effect, the semantic congruity effect, and the end effect. It also provides an explicit theoretical explanation of the processes underlying Dehaene's (1989) successful version of the discriminability model. We provide excellent fits to data from three representative experiments available in other papers, thus showing that the model can handle a variety of symbolic comparison data.

The distance effect and the semantic congruity effect are built into the model's structure. The end effect is imposed through the selection of the encoded stimuli. The codes fitted from the analyzed data suggest, as a rule of thumb, that the distance between codes of ordered symbols correlates with their relevance during the learning stage.

The model allows a straightforward generalization for the study of the RT distribution and incorrect answers. These are mainly originated in the noisy code values, but the RT variance may also have other sources. For example, the analysis of the linear syllogistic task data suggests that the variance across participants originates primarily in the variation across participants of the parameter $\lambda$, which controls the accrual rate.

The recursive model may be improved by integrating it with a learning device, as in the symbolic comparison model. Such an approach implies the development of a neural network with enough plasticity to provide code functions that change from linear to exponential or step shaped, depending on how the participant learned the ordering of the compared stimuli.

Our model may also be extended to describe RT and speed-accuracy tradeoff in comparison experiments with perceptual stimuli, possibly using signal detection theory, as has been done in similar models (Ratcliff, 1988; Usher \& McClelland, 2001). In the recursive model, the parameters that produce variations in accu- 
racy with varying demands for speed stress are $\lambda$ and $\Theta$. An increase in the effective recursive gain $\lambda$ determines an increase in speed and in incorrect answers. The bias $\Theta$ controls the interruption of the process at an arbitrary time. Furthermore, following Ratcliff (1978), the model can be naturally adapted to account for variability in memory strength for individual stimuli from trial to trial. Thus, we are confident that the recursive model may be extended to encompass perceptual comparisons and speed-accuracy tradeoff experiments. Nevertheless, the recursive model interpretation of the congruity effect has been tested only with symbolic comparisons. Petrusic and Baranski (1989) and Petrusic (1992) have shown that, with perceptual comparisons, this effect may be very robust and strong, especially if the compared stimuli are highly confusable. Therefore, these kinds of experiments could provide a different testing ground for the stopping conditions and exponential accrual of the recursive model.

\section{REFERENCES}

Ashby, F. G. (1983). A biased random walk model of two choice reaction times. Journal of Mathematical Psychology, 27, 277-297.

BANKS, W. P. (1977). Encoding and processing of symbolic information in comparative judgments. In G. H. Bower (Ed.), The psychology of learning and motivation (Vol. 11, pp. 101-159). New York: Academic Press.

BANKs, W. P., FujiI, M., \& Kayra-Stuart, F. (1976). Semantic congruity effects in comparative judgments of magnitudes of digits. Journal of Experimental Psychology: Human Perception \& Performance, 2, 435-447.

Buckley, P. B., \& Gillman, C. B. (1974). Comparisons of digits and dot patterns. Journal of Experimental Psychology, 103, 1131-1136.

Cohen, J. D., Dunbar, K., \& McClelland, J. L. (1990). On the control of automatic processes: A parallel distributed process account of the Stroop effect. Psychological Review, 97, 332-361.

Dehaene, S. (1989). The psychophysics of numerical comparison: A reexamination of apparently incompatible data. Perception \& Psychophysics, 45, 557-566.

DeHAENE, S. (1990). Is numerical comparison digital? Analogical and symbolic effects in two-digit number comparison. Journal of Experimental Psychology: Human Perception \& Performance, 16, 626-641.

HiNRICHS, J. V., YURKo, D. S., \& HU, J.-M. (1981). Two-digit number comparison: Use of place information. Journal of Experimental Psychology: Human Perception \& Performance, 7, 890-901.

HolyOAK, K. J. (1978). Comparative judgments in numerical reference points. Cognitive Psychology, 10, 203-243.

Jamieson, D. G., \& Petrusic, W. M. (1975). Relational judgments with remembered stimuli. Perception \& Psychophysics, 18, 373-378.

LAMING, D. R. J. (1968). Information theory of choice-reaction times. London: Academic Press.

Leth-Steensen, C., \& Marley, A. A. J. (2000). A model of response time effects in symbolic comparison. Psychological Review, 107, 62100.

LINK, S. W. (1990). Modeling imageless thought: The relative judgment theory of numerical comparisons. Journal of Mathematical Psychology, 34, 2-41.

Link, S. W., \& HeAth, R. A. (1975). A sequential theory of psychological discrimination. Psychometrika, 40, 77-105.

MARKs, D. F. (1972). Relative judgment: A phenomenon and a theory. Perception \& Psychophysics, 11, 156-160.

MoYer, R. S., \& Dumais, S. T. (1978). Mental comparison. In G. H. Bower (Ed.), The psychology of learning and motivation (Vol. 12, pp. 117-155). New York: Academic Press.

MOYER, R. S., \& LANDAUER, T. K. (1967). Time required for judgement of numerical inequality. Nature, 215, 1519-1520.

Petrusic, W. M. (1992). Semantic congruity effects and theories of the comparison process. Journal of Experimental Psychology: Human Perception \& Performance, 18, 962-986.

Petrusic, W. M., \& Baranski, J. V. (1989). Semantic congruity effects in perceptual comparisons. Perception \& Psychophysics, 45, 439452.

RATCLIfF, R. (1978). A theory of memory retrieval. Psychological Review, 85, 59-108.

RATCLIFF, R. (1988). Continuous versus discrete information processing: Modeling the accumulation of partial information. Psychological Review, 95, 238-255.

RATClifF, R., \& Rouder, J. N. (1998). Modeling response times for two-choice decisions. Psychological Science, 9, 347-356.

RATCLIFF, R., VAN ZANDT, T., \& MCKoon G. (1999). Connectionist and diffusion models of reaction time. Psychological Review, 106, 261300 .

Reynvoet, B., \& Brysbaert, M. (1999). Single-digit and two-digit Arabic numerals address the same semantic number line. Cognition, 72, 191-201.

Stone, M. (1960). Models for reaction time. Psychometrika, 25, 251260.

Trabasso, T. R., \& Riley, C. A. (1975). The construction and use of representations involving linear order. In R. L. Solso (Ed.), Information processing and cognition: The Loyola Symposium (pp. 381-410). Hillsdale, NJ: Erlbaum.

Trabasso, T. R., Riley, C. A., \& Wilson, E. G. (1975). The representation of linear order and spatial strategies in reasoning: A developmental study. In R. Falmagne (Ed.), Reasoning: Representation and process (pp. 201-230). Hillsdale, NJ: Erlbaum.

USHER, M., \& MCClELland, J. L. (2001). The time course of perceptual choice: The leaky, competing accumulator model. Psychological Review, 108, 550-592.

Welford, A. T. (1960). The measurements of sensory-motor performance: Survey and reappraisal of twelve years' progress. Ergonomics, 3, 189-230.

\section{NOTES}

1. Since the best $a, q$, and $b$ values have been evaluated to fit the RT curves, not all the parameters may be varied independently.

2 . This may be an oversimplified statement, since numbers such as $20,30,40$, and the like, probably have a distinct learning history and, therefore, their codes may be discriminated from those of other twodigit numbers. However, some authors have presented partial evidence against this argument (Dehaene, 1990; Reynvoet \& Brysbaert, 1999).

(Manuscript received December 2, 2001; revision accepted for publication June 16, 2003.) 